\title{
ENEA EUV Discharge Produced Plasma Source: Diagnostics, Characterization And Applications
}

\author{
Luca Mezi*, Sarah Bollanti, Francesco Flora and Amalia Torre \\ ENEA, APRAD Technical Unit, Radiation Sources Laboratory, Frascati, Italy \\ E-mail: luca.mezi@enea.it, sarah.bollanti@enea.it, \\ francesco.floralenea.it, amalia.torredenea.it
}

Extreme Ultraviolet (EUV) radiation (photon energy $h v=20-284 \mathrm{eV}$, wavelength $\lambda \simeq 4.4-$ $62 \mathrm{~nm}$ ) can be used to efficiently modify the structure of photonic materials, photoresists etc. In fact, the EUV short wavelength, its short penetration length in matter (typically few tens of nm) and the availability of high-reflectivity $(\geq 70 \%$ at $\lambda=13-15 \mathrm{~nm})$ normal-incidence multilayer mirrors allow high-spatial-resolution patterning on the aforementioned materials. This is leading to the development of the next generation lithography for micro-electronics. Consequently, EUV sources are a main object of study and they are largely exploited for metrology and tests on mirrors, innovative materials etc. Excluding large facilities like synchrotron machines, plasma sources are needed to efficiently generate EUV radiation.

An EUV discharge produced plasma source is operating at the ENEA Frascati Research Centre. The source characterization by means of various diagnostic tools will be reported after a brief description of its principle of operation. Finally, some source applications involving the treatment of both photonic materials and innovative photoresists will be illustrated.

First EPS Conference on Plasma Diagnostics - 1st ECPD,

14-17 April 2015

Villa Mondragone, Frascati (Rome) Italy

* Speaker. 


\section{Introduction}

An Extreme Ultraviolet (EUV) radiation source, based on a Discharge Produced Plasma (DPP), has been developed during a collaboration between ENEA and the Physics Department of the University of L'Aquila [1]. The EUV DPP source is now operating at ENEA, where proper modifications and upgrades have been carried out on the source in order to greatly improve its performances [2]. In the following, the ENEA EUV DPP source, its diagnostics and its characterization will be discussed. Finally, two source applications will be described.

\section{The ENEA EUV DPP source}

The DPP source is supplied by two electric discharges: an initial low-current pre-ionization one, followed by the high-current main discharge which leads to the high-temperature plasma formation [2]. The DPP source is optimized to emit EUV pulses in the $\lambda=10-20 \mathrm{~nm}$ range, being fed by xenon gas and a dipole magnet is used to deflect the ions emitted by the plasma. Briefly, the source working principle can be summarized as follows: low-pressure $(0.5-1.0 \mathrm{mbar}) \mathrm{Xe}$ gas, filling a short alumina capillary tube, is pre-ionized by using a low-current $(20-30 \mathrm{~A})$, long-duration $(10-20 \mu \mathrm{s})$ pulse; a low-inductance $50 \mathrm{nF}$ glycol cylindrical capacitor, charged to $18-20 \mathrm{kV}$, produces the main discharge $(10-11 \mathrm{kA}$ peak current, $240 \mathrm{~ns}$ base duration) in the pre-ionized gas; the resulting magnetic field $(>1 \mathrm{~T})$ pinches the plasma towards the capillary axis; the plasma resistance rises, thus its temperature can increase up to $30-40 \mathrm{eV}$; the hot plasma emits radiation peaked in the EUV region before relaxation and cooling.

The current source main operating parameters are reported in Tab. 1.

\begin{tabular}{|c|c|}
\hline Parameter & Operation value \\
\hline EUV emission band & $10-20 \mathrm{~nm}$ \\
\hline EUV pulse duration & $100 \mathrm{~ns}$ \\
\hline EUV source transverse dimension & $0.3 \mathrm{~mm}$ \\
\hline in-band pulse energy & $20 \mathrm{~mJ} / \mathrm{shot} / \mathrm{sr}$ \\
\hline repetition rate & $10 \mathrm{~Hz}$ \\
\hline pulse-to-pulse energy stability & $3-4 \% \mathrm{rms}$ \\
\hline usable emission cone & $\simeq 1 \mathrm{sr}$ \\
\hline continuous operation time & some hours \\
\hline debris emission & almost debris free \\
\hline
\end{tabular}

Table 1: ENEA EUV DPP source main operating parameters.

\section{Characterization and diagnostics}

Several High Voltage (HV) probes are used to measure the voltages on the main nodes of the source electrical circuit [2]. In particular, the resonant charging of the glycol capacitor which feeds the main discharge can be detected by using a proper calibrated resistive divider. The temporal 
evolution of this voltage is reported in Fig. 1, together with the Rogowski coil measured main discharge current and the emitted EUV radiation pulse.

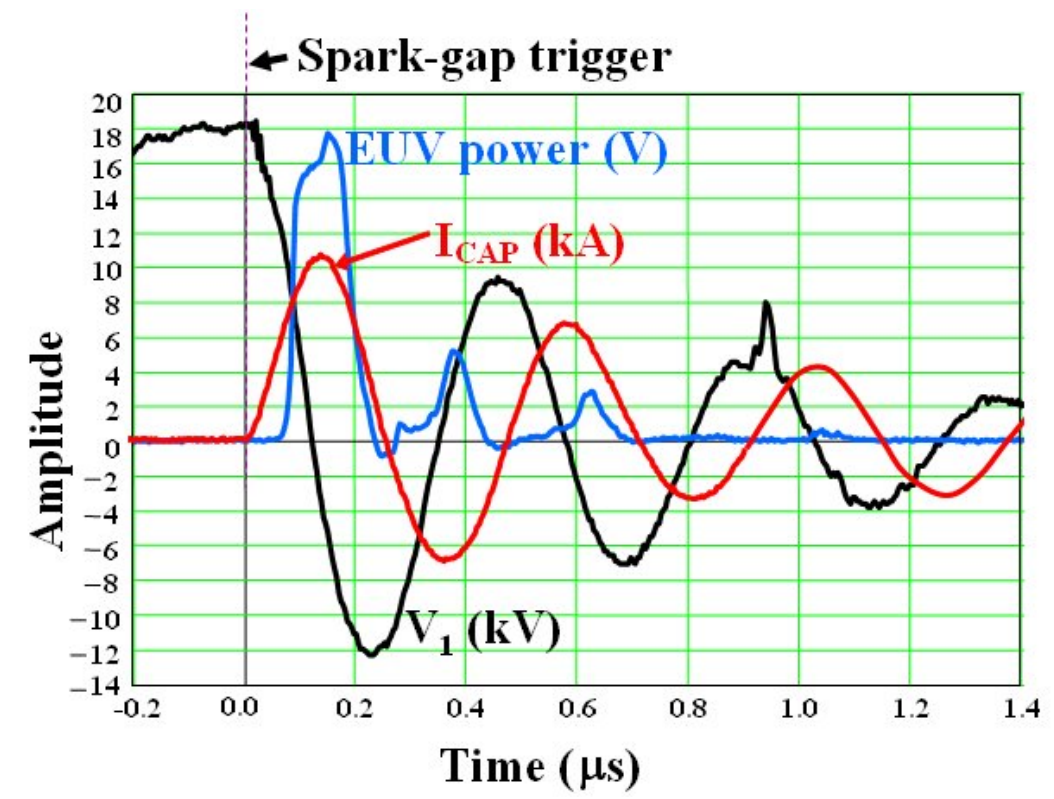

Figure 1: Temporal evolution of the HV $V_{1}$ on the capacitor which feeds the main discharge, of the main discharge current $I_{C A P}$ and the EUV pulse detected by a Zr-filtered PIN diode.

To select the $\lambda=10-20 \mathrm{~nm}$ wavelength range, a 150-nm-thickness zirconium filter [3] has been placed in front of the PIN diode. The EUV emission can occur after the plasma compression and its duration is shorter than that of the current.

In order to characterize the plasma evolution during the main discharge, it has been observed in the visible range by using a gated camera (DICAM-2, PCO Computer Optics GmbH, 1993) placed in front of the source at $1 \mathrm{~m}$ distance. The camera, equipped with a telephoto lens, monitored the plasma through a plexiglass window installed on the vacuum chamber which is in front of the source. The acquired images have been elaborated in false colours: in Figs. 2 and 3, the plasma images (in blue) are overlapped to the capillary tube and the HV electrode shapes (in red).

By using a gate time of $20 \mathrm{~ns}$ and changing the delay of the camera acquisition with respect to the main discharge starting time (only one image per shot is possible with this camera), the pinch effect is clearly demonstrated and a minimum plasma column size below $300 \mu \mathrm{m}$ in diameter has been detected as shown in Fig. 2.

Figure 2 shows that, after the collapse, the plasma column is reduced in size by more than one order of magnitude with respect to its starting value (which is the capillary internal diameter) and that the plasma shot-to-shot position stability is very good. The picture sequence also shows that the plasma collapse and successive relaxation temporally coincide with the EUV emission rise and fall, respectively, as expected. It is worth noticing that the visible emission power grows both during and after the EUV main pulse so that the optical density of a neutral filter placed in front of the camera had to be increased for longer delays in order to avoid the camera saturation. This can be related to the fact that the low-charge-state ions population, i. e. the responsible for visible light 

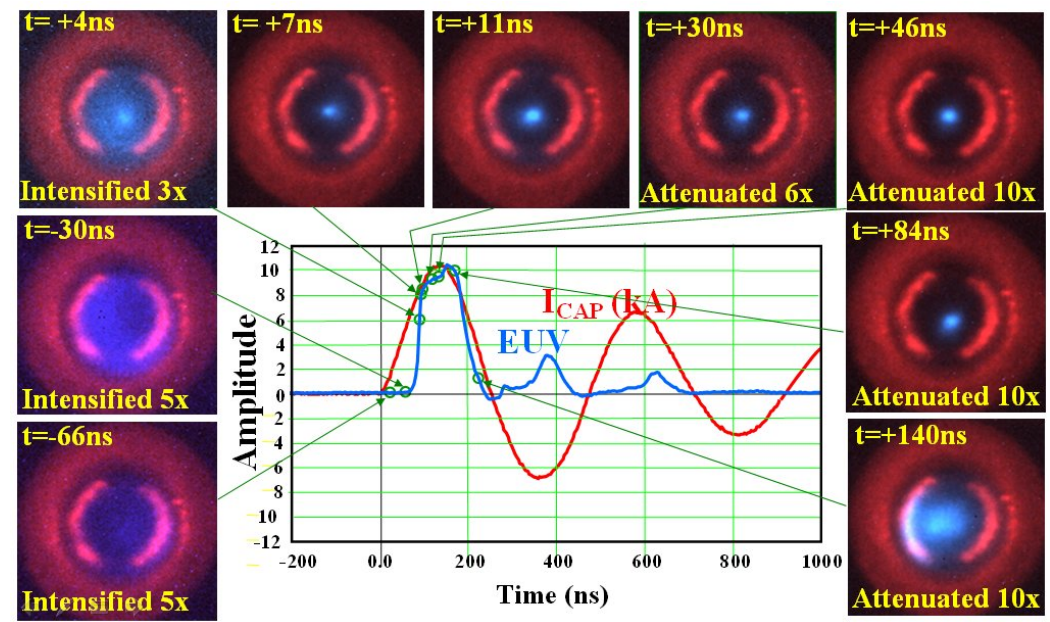

Figure 2: False colour images of plasma visible emission at different delay times $t$ from EUV rise (50\% of maximum). The inner red ring is the edge of the 3-mm-diameter HV electrode. The temporal position of each image with respect to the EUV power emission (blue curve) is indicated in the graph (green circles indicate the acquisition times of the gated camera). The red curve is the discharge current $I_{C A P}$.

emission, continuously grows during the main discharge.

The gated camera allowed also to analyze the discharge during the low-current pre-ionization phase, as shown in Fig. 3: at the current onset the discharge clearly occupies only a small portion of the capillary and only after about $2 \mu$ s it fills the whole tube. Hence, the main discharge triggering delay has been set a bit longer than such pre-ionization developing time. Figure 3 also shows that the onset of the pre-ionization discharge at each shot occurs in a random point of the capillary internal surface.
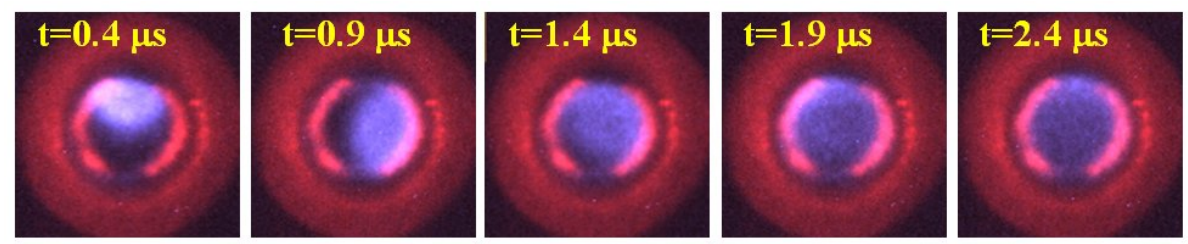

Figure 3: Sequence of false colour images of the discharge during the pre-ionization phase $(0.1 \mu$ s exposure time at a constant step of $0.5 \mu \mathrm{s}$, one image/shot). The delay time $t$ of each image with respect to the current onset is indicated.

The Gafchromic HD-V2 dosimetric film is another powerful tool that has been used to characterize the EUV DPP source. In Fig. 4a an example of the film using is reported: both the direct EUV radiation effect and a grazing reflection image are visible. Since the provided calibration curve is related to hard X-rays, the dosimetric film has been calibrated in the $\lambda=10-20 \mathrm{~nm}$ EUV region by using the DPP source, as shown in Fig. 4b. In order to fit the experimental data, the following formula for a photografic thick film has been used:

$$
g=10^{-\left[\frac{D_{S}}{2} \ln \left(1+\frac{F}{F_{S}}\right)\right]}
$$


where $g$ is the grey level, $D_{s}$ is the saturation optical density, $F$ is the EUV fluence and $F_{s}$ is the EUV saturation fluence. The very good agreement between the experimental data and the best-fit curve, as shown in Fig. 4b, allows also a quantitative use of the dosimetric film in the EUV region.

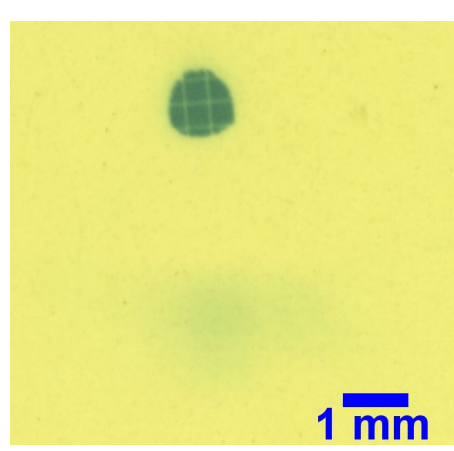

a)

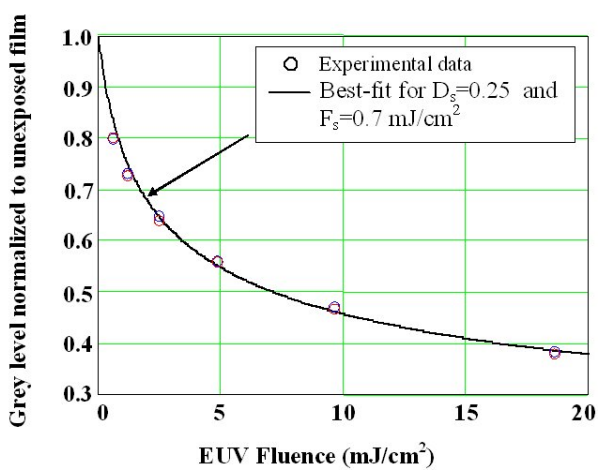

b)

Figure 4: Gafchromic HD-V2 dosimetric film: a) exposed through a 1-mm-diameter pin-hole (covered by a $150 \mathrm{~nm}$ thick $\mathrm{Zr}$ filter supported by a $\mathrm{Ni}$ wires mesh) at $17 \mathrm{~mm}$ from the film, $56 \mathrm{~cm}$ from the plasma and $\simeq 10^{\circ}$ off-axis; the upper disk is given by the direct radiation, while the lower soft halo is given by grazing reflections on the vacuum chamber ceiling; b) EUV calibration curve, experimental grey levels (red channel, acquired by a quadratic sensitivity 16 bits scanner) vs EUV fluence (circles) and the best-fit function.

\section{EUV DPP source applications}

Among the various applications, we mention the radiography of an aluminum filter imaged on a lithium fluoride (LiF) crystal. EUV generates stable colour centres in LiF [4] which can be observed by using a fluorescence microscope, as shown in Fig. 5. The sample, placed in the vacuum chamber at $10 \mathrm{~cm}$ from the plasma, has been exposed to 8000 shots of the DPP source in order to detect the filter irregularities. Since most of the EUV radiation is emitted by the DPP source at $\lambda<17 \mathrm{~nm}[1,2]$, while the Al filter mostly transmits EUV radiation for $\lambda>17 \mathrm{~nm}$ [3], a low EUV dose $\left(\simeq 0.1 \mathrm{~J} / \mathrm{cm}^{2}\right)$ reached the LiF, in spite of the large number of shots.

Another application is the EUV direct exposure of novel photoresists in the framework of a project, funded by the CARIPLO Foundation, which involves ENEA and the Universities of Pavia and Padova. An example of EUV exposure of a resist synthesized at the Padova University is shown in Fig. 6.

\section{Concluding remarks}

An EUV source, based on a discharge produced plasma, is operating at the ENEA Frascati Laboratories. The source allows reliable and clean direct EUV exposures over a wide cone of emission. The main operating parameters of the source are reported in Tab. 1. Several diagnostics have been exploited to characterize the EUV plasma source: electrical probes, a gateable CCD and a dosimetric film which has been also calibrated in the $10-20 \mathrm{~nm}$ wavelength range. The ENEA EUV DPP source is available also for other experiments. 


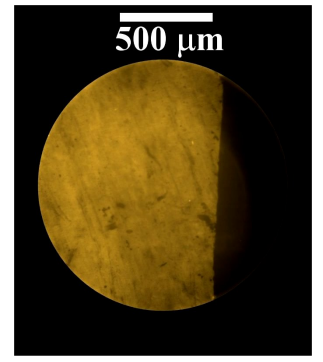

$10 \mathrm{x}$

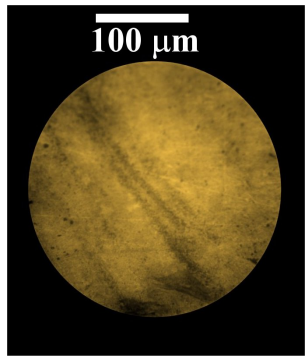

$50 \mathrm{x}$

Figure 5: Radiography of an $\mathrm{Al}$ filter. A LiF crystal, covered by a $0.8 \mu \mathrm{m}$ thick $\mathrm{Al}$ membrane, has been exposed to 8000 shots $\left(\simeq 2 \mathrm{~J} / \mathrm{cm}^{2}\right)$ at $10 \mathrm{~cm}$ from the DPP source and observed by using a fluorescence microscope at different magnification levels. The filter irregularities are visible as luminescent patterns.

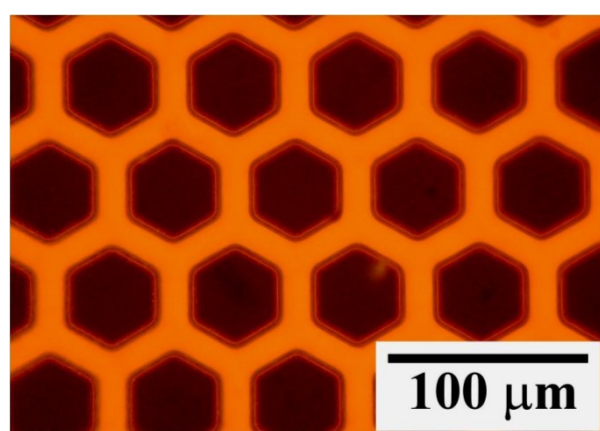

a)

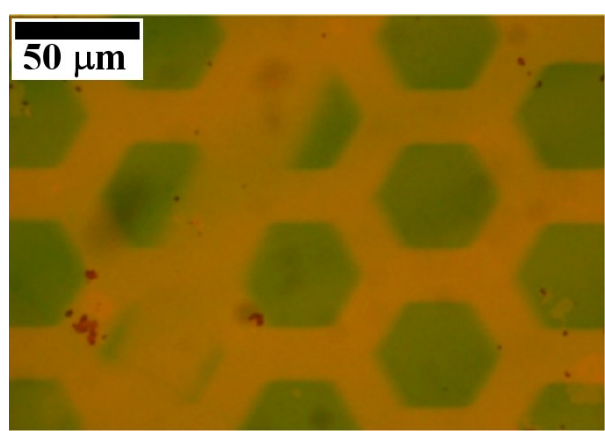

b)

Figure 6: Photoresist EUV direct exposure: a) copper grid ( 400 holes/inch) used as contact mask; b) same mask reproduced on a Titania "in-situ" loaded photoresist. Exposure: $150-\mathrm{nm}-\mathrm{Zr}$ filter, $10 \mathrm{~cm}$ from plasma, 4000 shots $\left(\simeq 0.5 \mathrm{~J} / \mathrm{cm}^{2}\right.$ on photoresist). The shadow of a Ni wire supporting the $\mathrm{Zr}$ filter is visible.

\section{Acknowledgments}

The authors thank the CARIPLO Foundation for funding the project: "New materials for direct nanopatterning and nanofabrication by EUV and soft X-rays exposures" (04/2013-12/2015).

\section{References}

[1] P. Zuppella, et al., Spectral enhancement of a Xe-based EUV discharge plasma source, Plasma Sources Sci. Tech. 18 (2009) 025014.

[2] L. Mezi, F. Flora, La sorgente DPP di radiazione nell'estremo ultravioletto a scarica elettrica in gas rarefatto, ENEA Technical Report RT/2012/15/ENEA, http://opac.bologna.enea.it:8991/RT/2012/2012_15_ENEA.pdf.

[3] B. L. Henke et al. http://henke.lbl.gov/optical_constants.

[4] G. Baldacchini et al., Soft x-ray submicron imaging detector based on point defects in LiF, Rev. Sci. Instr., 76, 2005, 113104-1-12. 